\title{
Challenges and trends of changing from hover to forward flight for a converted hybrid fixed wing VTOL UAS from automatic flight control system perspective
}

\begin{abstract}
Capabilities of fixed-wing UAS to perform missions have been well studied and documented today. And so has for multirotor drones. It is also well accepted that both platforms have their strengths and limitations during operation. For this reason, one suggestion to address those limitations is to create a 'hybrid' UAS which will incorporate the best capabilities of both types of aircraft. This paper proposes retaining the best features of an already flying long endurance fixed wing UAS and then incorporate a vertical takeoff capability. The paper looks at the challenges and issues of transitioning from hovering to forward flight. From the 3 strategies proposed, Strategy 1 suggests to increase the speed of rotation of the 2 aft rotors therefore, causing the aircraft to pitch forward, hence generating a forward speed from hovering. For Strategy 2 during hovering, all 4 rotors are spinning at the same speed hence generating equal balanced thrust upwards for the hybrid aircraft. Then, the aircraft main petrol engine is set to full throttle to generate forward thrust to commence flight forward. For Strategy 3, the 4 rotors are tasked as in Strategy 2 but once a safe altitude is reached, the 4 rotors are disengaged to let the aircraft nose drop forward to commence a controlled dive. At the same time, the aircraft main petrol engine is set to full throttle to gain airspeed to continue with normal forward flight. The paper concludes with a brief description of the role of automatic flight control systems responsible for regulating the transition phase, setting up the future work for future work on modelling, simulation, fabrication and flight testing.
\end{abstract}

Keyword: Dual mode UAS; Multirotor; Drones; VTOL UAS; VTOL changing speeds; Smart agriculture; Rotor strategy 